\title{
Motion of vortex lines in quantum mechanics*
}

\author{
Iwo Bialynicki-Birula ${ }^{\dagger 1,4}$, Zofia Bialynicka-Birula ${ }^{2,3}$, and Cezary Śliwa ${ }^{1,3}$ \\ Center for Theoretical Physics ${ }^{1}$, Institute of Physics ${ }^{2}$, College of Science ${ }^{3}$, \\ Al. Lotników 32/46, 02-668 Warsaw, Poland and \\ Institute of Theoretical Physics ${ }^{4}$, Warsaw University, Hoża 69, 00-681 Warsaw, Poland
}

\begin{abstract}
Exact analytic solutions of the time dependent Schrödinger equation are produced that exhibit a variety of vortex structures. The qualitative analysis of the motion of vortex lines is presented and various types of vortex behavior are identified. Vortex creation and annihilation and vortex interactions are illustrated in the special cases of the free motion, the motion in the harmonic potential, and in the constant magnetic field. Similar analysis of the vortex motions is carried out also for a relativistic wave equation.
\end{abstract}

\section{INTRODUCTION}

Vortices have been a source of fascination since time immemorial. Empedocles, Aristotle [1], and Descartes [2] tried to explain the formation of the Earth, its gravity, and the dynamics of the whole solar system as due to primordial cosmic vortices. Lord Kelvin [3] envisaged atoms as vortex rings. Modern science of hydrodynamic vortices began with the fundamental work of Helmholtz [4] on the theory of vorticity. The present knowledge of vortex dynamics in fluid mechanics is best summarized in a recent monograph by Saffman [5].

In our paper we shall be concerned with vortices not in ordinary fluids but in a peculiar fluid - the probability fluid - that can be associated with the wave function of a quantum system. Vortices of that type have been studied in the past in connection with superfluidity, superconductivity, and recently in connection with Bose-Einstein condensation $[6$ 9]. The main tool in the study of these phenomena is the Ginzburg-Landau (Gross-Pitaevskii) equation. The nonlinear character of this equation greatly complicates the analysis of time evolution of the vortices, especially in three dimensions. There are no exact analytic solutions of this equation with nontrivial vortex structure. One must resort either to approximations which can reveal only some features of the vortex motion (as, for example, in the early work of Fetter 10,11]) or to extensive numerical calculations (cf., for example, 12 ). In the present paper we use the standard time-dependent Schrödinger equation to investigate the motion of vortex lines embedded in the probability fluid of a quantum particle. The linearity of this equation enables us to obtain a variety of exact, time-dependent analytic solutions for the wave functions that describe the probability flow with one, two or more moving vortex lines. We are also able to study the motion of vortex lines in the presence of ex- ternal forces. Our analysis of many special cases makes it possible to discover certain general phenomena such as the vortex switchover and the vortex creation and annihilation. The important conclusion of our analysis is that quantum effects play a crucial role in those phenomena. We believe that our analysis captures the essential features of quantum vortex dynamics that are common to many different situations. In particular, our analysis also extends to the relativistic domain.

We have to admit that vortex lines associated with wave functions are rather elusive objects. They might be connected with physical reality in two ways. First, the vortex lines can appear only at those places where the wave function is equal to zero; the vanishing of the probability density definitely has observable consequences. Second, the wave function of a macroscopically populated state of many bosons acquires a direct physical interpretation; vortex lines in this case may become directly observable as, for example, in BEC 13, 14 .

\section{VORTEX LINES IN THE HYDRODYNAMIC FORMULATION OF WAVE MECHANICS}

In quantum theory vortex lines arise in the hydrodynamic formulation of wave mechanics. This formulation originally due to Madelung [15] (see also a review article [16]) employs the hydrodynamic variables to describe the flow of quantum mechanical probability for a single particle. The probability density $\rho(\mathbf{r}, t)$ and the velocity field $\mathbf{v}(\mathbf{r}, t)$ are connected with the wave function $\psi(\mathbf{r}, t)$ through the formulas

$$
\begin{gathered}
\psi(\mathbf{r}, t)=R(\mathbf{r}, t) \exp [i S(\mathbf{r}, t) / \hbar] \\
\rho(\mathbf{r}, t)=|\psi(\mathbf{r}, t)|^{2}=R^{2}(\mathbf{r}, t),
\end{gathered}
$$

\footnotetext{
${ }^{*}$ To appear in Physical Review A.

†email: birula@cft.edu.pl
} 


$$
\begin{aligned}
\mathbf{v}(\mathbf{r}, t) & =\frac{1}{m} \frac{\operatorname{Re}\left[\psi^{*}(\mathbf{r}, t)(-i \hbar \nabla-e \mathbf{A}) \psi(\mathbf{r}, t)\right]}{|\psi(\mathbf{r}, t)|^{2}} \\
& =\frac{1}{m}(\nabla S(\mathbf{r}, t)-e \mathbf{A}(\mathbf{r}, t)),
\end{aligned}
$$

where $\mathrm{m}$ is the mass of the particle and $\mathbf{A}(\mathbf{r}, t)$ is the electromagnetic vector potential. The gradient term $\nabla S$ in (3) does not contribute to vorticity. Thus, the bulk vorticity $\nabla \times \mathbf{v}(\mathbf{r}, t)$ in the probability fluid is completely determined by the magnetic field except at the points where the phase $S$ is singular. This may occur only at the points where the wave function vanishes. The vanishing of the complex wave function, in turn, gives two conditions: the vanishing of two real functions. Each condition defines a two-dimensional surface. The intersection of these two surfaces defines a line. Thus, in addition to the given distribution of vorticity in the bulk of the probability fluid, that is uniquely determined by the magnetic field, we may also have isolated vortex lines embedded in the fluid. Along these lines the vorticity has a two-dimensional Dirac delta-function singularity in the plane perpendicular to each vortex line. It is the dynamics of these isolated vortex lines that will be the subject of our study. In this context we shall identify vortex lines with line vortices.

To ensure that the wave function is single valued, the strength of every vortex as measured by the circulation $\Gamma$ along any closed contour $C$ encircling the vortex line,

$$
\Gamma=\oint_{C} d \mathbf{l} \cdot \mathbf{v}(\mathbf{r}, t)
$$

must be quantized:

$$
\Gamma=\frac{2 \pi \hbar}{m} n, \quad n=0, \pm 1, \pm 2, \ldots
$$

As one approaches the vortex line the velocity $\mathbf{v}(\mathbf{r}, t)$ must tend to infinity in order to satisfy the quantization condition (5).

The circulation is conserved during the time evolution 17,18 (in full correspondence with the Helmholtz-Kelvin law for ordinary nonviscous fluids [19]). The creation of vortex lines may proceed through two different scenarios: they may either appear in the form of a closed vortex line that springs from a point (Fig. 11) or they can be created in pairs of opposite circulation (Fig. 2).

The hydrodynamic formulation can also be introduced in wave mechanics of a spinning particle governed by the Pauli equation 18,20,21.

Despite all the formal similarities between the hydrodynamics of classical fluids and the hydrodynamic formulation of quantum mechanics, there is a fundamental difference between the properties of line vortices in these two cases. In classical hydrodynamics, as pointed out by Saffman [5], p. 36, "the line vortex is a useful kinematic concept, but it does not exist as a sensible dynamical limit". In quantum mechanics the line vortices occur in exact solutions of the Schrödinger equation without any limiting procedure. The fact that the velocity becomes infinite as one approaches the line vortex is acceptable, because it is only the probability and not any real matter that flows with that velocity.

\section{THE ANATOMY OF A VORTEX LINE}

The commonly known examples of vortex lines are those embedded in the wave functions that have a fixed value $\hbar m_{z}$ of the $z$-component of angular momentum (eg., the spherical harmonics). These vortex lines run from $-\infty$ to $\infty$ along the $z$-axis and have the circulation $\hbar m_{z} / m$. Usually they are stationary, due to angular momentum conservation. In addition to straight vortex lines, we can also easily produce stationary vortex rings by superposing wave functions with the same energy but different angular momenta. The simplest example is the superposition (with a $\pi / 2$ phase difference) of the electronic $\psi_{200}$ and $\psi_{210}$ states in hydrogen. The vortex ring is formed in $z=0$ plane and has the radius of two Bohr radii.

In our study we shall discuss a broader class of vortices that, in general, do not exhibit cylindrical symmetry even when they form a straight line. Let us consider an arbitrary vortex line $\boldsymbol{\xi}(s)$ parametrized by the length $s$ along the line. A typical quantum vortex is associated with the line of first order zeros of the wave function. Higher order zeros will, in general, lead to vortices that carry more that one unit of vorticity. The local properties of such a vortex at a point $\boldsymbol{\xi}(s)$ are determined by the behavior of the wave function near this point

$$
\psi(x, y, z) \approx(\mathbf{r}-\boldsymbol{\xi}(s)) \cdot \nabla \psi(\boldsymbol{\xi}(s)),
$$

where we have kept only the lowest terms in the Taylor expansion. Since quantum vortices have zero thickness, this approximation is always valid sufficiently close to the vortex line. At each point on the vortex line let us define a complex vector $\mathbf{w}(s)$ - the gradient of the wave function

$$
\mathbf{w}(s)=\left.\nabla \psi(\mathbf{r})\right|_{\mathbf{r}=\boldsymbol{\xi}(s)} .
$$

We shall show that this vector describes the basic properties of the vortex. Let us note that since the derivative of the wave function along the vortex line with respect to the length parameter $s$ is zero, the real and the imaginary parts of the vector $\mathbf{w}(s)$ lie in the plane perpendicular to the tangent vector $\mathbf{t}(s)=d \boldsymbol{\xi}(s) / d s$,

$$
\frac{d \psi(\boldsymbol{\xi}(s))}{d s}=\mathbf{t}(s) \cdot \mathbf{w}(s)=0 .
$$

The general complex vector $\mathbf{w}$ has 4 real components, but only 2 parameters contain the information about the vortex, since the multiplication of the wave function by a complex number does not change, of course, the velocity 
field. Indeed, the velocity field $\mathbf{v}(x, y, z)$ calculated for the approximate wave function (6),

$$
\begin{aligned}
\mathbf{v}(x, y, z) & =\frac{\hbar}{2 m i}\left(\frac{\mathbf{w}}{\mathbf{w} \cdot \mathbf{r}}-\frac{\mathbf{w}^{*}}{\mathbf{w}^{*} \cdot \mathbf{r}}\right) \\
& =\frac{\hbar}{2 m i} \frac{\mathbf{r} \times\left(\mathbf{w} \times \mathbf{w}^{*}\right)}{|\mathbf{w} \cdot \mathbf{r}|^{2}}
\end{aligned}
$$

is a homogeneous function of $\mathbf{w}$. Therefore, $\mathbf{v}$ does not change when $\mathbf{w}$ is multiplied by any complex number. For simplicity, we have chosen the origin of the coordinate frame at the point $\boldsymbol{\xi}(s)$. Only in the special case, when the real and imaginary part of $\mathbf{w}$ are mutually orthogonal and of equal length, the velocity lines follow circles in the plane orthogonal to the vortex line. In all other cases the vortex is "squeezed"; the velocity lines follow ellipses. In the degenerate case, when the real part and the imaginary part of $\mathbf{w}$ are parallel, the ellipse is squeezed into a line and the velocity disappears. The vector $\mathbf{w}$ will, in general, vary as we move along the vortex line. However, one may check by a direct calculation that the circulation (蛋) calculated for the velocity field (9) does not depend on $\mathbf{w}$ and is equal to $\pm 2 \pi \hbar / m$ (the sign depends on the orientation of the contour $C$ ). Higher values of the circulation are obtained when the wave function has zeros of the higher order but the typical case, of course, is the first order zero.

The above analysis shows that the appearance of vortex lines in wave mechanics does not require that some special conditions be met. On the contrary, a generic wave function has vortex lines. The vanishing of the real and the imaginary part of the wave function defines a line. The Taylor expansion of the wave function around the point lying on this line defines the complex vector $\mathbf{w}$ that determines the local structure of the vortex.

The vector $\mathbf{w}$ not only determines the velocity field but also plays a crucial role in determining the motion of the vortex line itself. The velocity $\mathbf{u}=d \boldsymbol{\xi}(s, t) / d t$ of a point $\boldsymbol{\xi}(s, t)$ lying on the vortex line can be obtained from the condition

$$
d \psi(\boldsymbol{\xi}(s, t), t) / d t=\mathbf{u} \cdot \mathbf{w}+\partial \psi / \partial t=0 .
$$

The vector $\mathbf{u}$ is defined only up to a vector parallel to the vortex line and it can be determined by solving Eq.(10):

$$
\mathbf{u}=\frac{\mathbf{w} \times \mathbf{w}^{*}}{\left|\mathbf{w} \times \mathbf{w}^{*}\right|^{2}} \times\left(\frac{\partial \psi^{*}}{\partial t} \mathbf{w}-\frac{\partial \psi}{\partial t} \mathbf{w}^{*}\right) .
$$

Using the Schrödinger equation,

$$
i \hbar \partial_{t} \psi(\mathbf{r}, t)=\left[-\frac{\hbar^{2}}{2 m} \Delta+V(\mathbf{r})\right] \psi(\mathbf{r}, t),
$$

we obtain

$$
\mathbf{u}=\frac{\hbar}{2 m i} \frac{\mathbf{w} \times \mathbf{w}^{*}}{\left|\mathbf{w} \times \mathbf{w}^{*}\right|^{2}} \times\left(\mathbf{w} \Delta \psi^{*}+\mathbf{w}^{*} \Delta \psi\right) .
$$

Let us notice that the potential term $V$ does not appear in the formula for the velocity $\mathbf{u}$ since the wave function vanishes on the vortex line. The motion of the vortex line at a given point is completely determined by the local properties of the wave function: its gradient and its Laplacian at this point. There is no direct relation between the velocity $\mathbf{u}$ of the vortex line and the velocity $\mathbf{v}$ of the probability fluid.

\section{GENERAL METHOD OF GENERATING VORTEX LINES}

Let us choose the initial condition for the solution of the time-dependent Schrödinger equation in the form

$$
\phi_{\mathbf{k}}(\mathbf{r})=\exp (i \mathbf{k} \cdot \mathbf{r}) \phi_{0}(\mathbf{r}) .
$$

By differentiating $\phi_{\mathbf{k}}$ with respect to the components of the vector $\mathbf{k}$ any number of times we obtain new wave functions. Each differentiation brings down a component of the position vector and in this manner we may generate an arbitrary complex polynomial that multiplies the initial wave function. Carrying out the differentiations, adding the results with appropriate complex coefficients, and setting $\mathbf{k}=0$ at the end, we arrive at the expression for the initial wave function of the form

$$
\left(W_{R}(\mathbf{r})+i W_{I}(\mathbf{r})\right) \phi_{0}(\mathbf{r}),
$$

where $W_{R}$ and $W_{I}$ are real polynomials in the three variables $x, y$, and $z$.

The conditions $W_{R}(\mathbf{r})=0$ and $W_{I}(\mathbf{r})=0$ define two two-dimensional surfaces in the three-dimensional space. When these surfaces intersect, they define a line. This will be a vortex line if the circulation around it is different from zero, which is the generic case. This procedure leads to wave functions that have initially vortex lines of almost arbitrary shape and topology embedded in them. In other words, the wave function $\phi_{\mathbf{k}}$ serves as the generating function for vortex lines. In practice we are facing technical limitations; for higher order polynomials the analysis becomes very cumbersome. However, if the polynomial $W_{R}+i W_{I}$ is constructed as a product of some simple polynomials in the form

$$
\begin{aligned}
W_{R}(x, y, z) & +i W_{I}(x, y, z)=\left(W_{R}^{\prime}(x, y, z)+i W_{I}^{\prime}(x, y, z)\right) \\
& \times\left(W_{R}^{\prime \prime}(x, y, z)+i W_{I}^{\prime \prime}(x, y, z)\right) \ldots, \quad(16)
\end{aligned}
$$

then the initial wave function is easily analyzed: it has several vortex lines, each corresponding to one of the factors in (16). Most of our discussion will be restricted to second degree polynomials which already lead to a plethora of different shapes and behaviors.

The time evolution of vortex lines that were embedded in the initial wave function is determined, of course, by the Schrödinger equation. The advantage of using the generating function (14) is that once we find the timedependent solution $\psi_{\mathbf{k}}(\mathbf{r}, t)$ satisfying the initial condition 


$$
\psi_{\mathbf{k}}(\mathbf{r}, t=0)=\phi_{\mathbf{k}}(\mathbf{r})
$$

we may use it to generate all solutions that at $t=0$ have the form (15). Since the vector $\mathbf{k}$ does not appear in the Schrödinger equation, the differentiations with respect to its components do not interfere with the time evolution: $\mathbf{k}$-derivatives of the time-dependent wave function are also solutions of the Schrödinger equation. Carrying out the differentiations with respect to the components of $\mathbf{k}$ on the time dependent function $\psi_{\mathbf{k}}(\mathbf{r}, t)$, we shall obtain the time evolution of vortex lines from the time dependence of the polynomials $W_{R}(\mathbf{r}, t)$ and $W_{I}(\mathbf{r}, t)$ introduced in the formula (15).

Obviously, wave functions are not restricted to those that have only a finite number of vortex lines described by a polynomial prefactor (15). For example, replacing the sum of polynomials by the sum of trigonometric functions $\cos (x / a)+i \cos (y / b)$ leads to an infinite "forest" of vortex lines pointing in the $z$-direction.

\section{VORTEX LINES FOR A FREELY MOVING PARTICLE}

In this Section we shall describe the time evolution of vortex lines for a freely moving particle. The simplest solution of the corresponding time-dependent Schrödinger equation, that has at $t=0$ the form (14), corresponds to $\phi_{0}(\mathbf{r})=1$,

$$
\psi_{\mathbf{k}}(\mathbf{r}, t)=\exp (i \mathbf{k} \cdot \mathbf{r}) \exp \left(-i \hbar \mathbf{k}^{2} t / 2 m\right) .
$$

Such a plane-wave solution is not square-integrable but that can easily be corrected by multiplying $(18)$ by a slowly varying envelope without affecting significantly the dynamics of vortex lines. We shall return to this problem at the end of this Section.

Let us begin with the simplest solution that initially has one rectilinear vortex described by the wave function $(x \cos \chi+i y \sin \chi) \exp (i \mathbf{k} \cdot \mathbf{r})$. The parameter $\chi$ measures the degree of squeezing of the velocity field. Differentiating the wave function (18) with respect to $k_{x}$ and $k_{y}$ we obtain the wave function with a vortex line. Since the parameters $k_{x}, k_{y}$, and $k_{z}$ in (18) can be interpreted as the components of the particle momentum, $\hbar \mathbf{k}=\mathbf{p}$, it is instructive not to set $\mathbf{k}$ to zero but keep it finite after the differentiation. This will result in a free motion of the whole vortex line with the velocity $\mathbf{v}=\hbar \mathbf{k} / m$ :

$$
(x(t) \cos \chi+i y(t) \sin \chi) \psi_{\mathbf{k}}(\mathbf{r}, t)
$$

where $x(t)=x-v_{x} t$ and $y(t)=y-v_{y} t$. Throughout this paper $x(t), y(t)$ and $z(t)$ will always denote a free classical motion with constant velocity. Eq. (19) means that the vortex line riding on the plane wave is moving uniformly, without changing its shape, with the speed determined by the particle momentum. This was to be expected as a consequence of the Gallilean invariance of the theory. The same free motion of a single vortex line was found in the nonlinear Schrödinger equation [10].

Our next example of a vortex is a ring of radius $R$. It can be obtained, for example, by cutting a cylinder by a plane:

$$
W_{R}(x, y, z)=x^{2}+y^{2}-R^{2}, \quad W_{I}(x, y, z)=a z .
$$

The ratio of the two dimensional parameters $R$ and $a$ determines the degree of squeezing of the vortex, $\tan \chi=$ $a / 2 R$. The motion of this vortex is described by the time-dependent wave function

$$
\begin{aligned}
\psi^{C}(\mathbf{r}, t) & =\left[x(t)^{2}+y(t)^{2}-R^{2}+i a\left(z(t)+\frac{2 \hbar t}{m a}\right)\right] \\
& \times \psi_{\mathbf{k}}(\mathbf{r}, t) .
\end{aligned}
$$

The quantum correction (the term $2 \hbar t / m a$ ) to the classical vortex motion that appears here amounts only to a change of the vortex velocity in the direction of the cylinder axis. In this example, the vortex ring in the form of a circle has been produced by intersecting a cylinder and a plane. However, there are many other possibilities, even with the use of only simple surfaces. It is easy to obtain at $t=0$ the same shape of the vortex line choosing different wave functions. The motion of these vortices may change dramatically, when one pair of surfaces is replaced by a different one. The circular vortex formed by intersecting a cylinder with a plane, as shown above, moves uniformly along the cylinder axis. On the other hand, the same vortex produced at $t=0$ by a sphere and a plane will contract to a point and disappear. The appropriate wave function has the form

$$
\begin{aligned}
\psi^{S}(\mathbf{r}, t) & =\left[x(t)^{2}+y(t)^{2}+z(t)^{2}-R^{2}+i a\left(z(t)+\frac{3 \hbar t}{m a}\right)\right] \\
& \times \psi_{\mathbf{k}}(\mathbf{r}, t) .
\end{aligned}
$$

The circle disappears, i.e., the vortex is annihilated, at the time $t_{a}=m a R / 3 \hbar$. Extending our analysis to negative values of $t$, we see that this vortex was created at the time $t_{c}=-m a R / 3 \hbar$ (Fig. 1). A circular vortex obtained by intersecting a paraboloid with a plane has again a different motion: after it is created, it keeps expanding and moving along the $z$-axis to infinity. Other motions of a circular vortex line can also be obtained by choosing a second quadratic surface, instead of a plane. In all these cases the motion of the vortex ring has two components. One is the free motion with the velocity $\mathbf{v}$ and the other is a quantum correction that may lead to the vortex annihilation. We would like to stress that the creation and annihilation of vortex rings are of a purely quantum origin. These phenomena are not found in the studies of motion of the vortex ring based on the semiclassical approximation [10,11].

The creation and annihilation of vortex rings at a point, shown in Fig. 1, does not contradict the Helmholtz-Kelvin theorem on the conservation of circulation. This theorem, obviously, holds only inside the 
fluid but the vortex creation and the vortex annihilation occur at the points where the fluid density vanishes.

The mutual interaction between vortex lines can be seen in the motion of two vortex lines. The initial wave function containing two arbitrary rectilinear vortices has the product form

$$
\phi_{\mathbf{k}}(\mathbf{r})=\left(\mathbf{w}_{1} \cdot\left(\mathbf{r}-\mathbf{r}_{1}\right)\right)\left(\mathbf{w}_{2} \cdot\left(\mathbf{r}-\mathbf{r}_{2}\right)\right) \exp (i \mathbf{k} \cdot \mathbf{r}) .
$$

The corresponding time-dependent wave function, obtained by our method is

$$
\begin{aligned}
\psi(\mathbf{r}, t) & =\left[\left(\mathbf{w}_{1} \cdot\left(\mathbf{r}(t)-\mathbf{r}_{1}\right)\right)\left(\mathbf{w}_{2} \cdot\left(\mathbf{r}(t)-\mathbf{r}_{2}\right)\right)+\frac{i \hbar t}{m} \mathbf{w}_{1} \cdot \mathbf{w}_{2}\right] \\
& \times \psi_{\mathbf{k}}(\mathbf{r}, t) .
\end{aligned}
$$

Thus, the two vortices become, in general, entangled by the quantum correction term: the wave function is not a product of factors describing two separate vortices. The coupling between the two vortices is due to the interaction between the vortices and it vanishes only when $\mathbf{w}_{1} \cdot \mathbf{w}_{2}=0$. The time evolution exhibiting the phenomenon of a switchover of the two vortex lines is shown in Fig. 3. To simplify the calculations we assumed that both vortices are nonsqueezed. The coordinate system is chosen in such a way that at $t=0$ the vortex lines lie in the $z= \pm a$ planes with the angle between them equal to $2 \varphi$. With this choice, the time-dependent wave function can be written in the form

$$
\psi(\mathbf{r}, t)=\left[W_{1} W_{2}-(2 i \hbar t / m) \sin ^{2} \varphi\right] \psi_{\mathbf{k}}(\mathbf{r}, t),
$$

where the two polynomials $W_{1}$ and $W_{2}$,

$$
\begin{aligned}
& W_{1}(\mathbf{r}, t)=x(t) \cos \varphi+y(t) \sin \varphi+i(z(t)+a), \\
& W_{2}(\mathbf{r}, t)=x(t) \cos \varphi-y(t) \sin \varphi+i(z(t)-a),
\end{aligned}
$$

describe the motion of both vortex lines separately, whereas the last term in the square bracket is responsible for their interaction. Only when $\varphi=0$ the vortex lines remain unchanged during the time evolution. This behavior is different from what can be found in non-compressible fluids [19]. The quantum interaction between the vortex lines is the strongest when the lines are antiparallel (i.e. the two vortices have opposite circulation, $\varphi=\pi / 2)$. In this case the vortex lines retain their direction but the distance between them decreases and at time $t_{a}=m a^{2} / \hbar$ the vortex lines collide and annihilate. The same wave function describes at $t_{c}=-m a^{2} / \hbar$ the creation of two rectilinear vortices of opposite circulation. These processes are shown in Fig. 2․

We shall now replace the plane wave solution of the Schrödinger equation, corresponding to $\phi_{0}(\mathbf{r})=$ 1 , by a normalizable Gaussian wave packet $\phi_{0}(\mathbf{r})=$ $\exp \left(-\mathbf{r}^{2} / 2 l^{2}\right)$. This will give us square integrable solutions with vortex lines. The generating function for such solutions has the form:

$$
\psi_{\mathbf{k}}^{G}(\mathbf{r}, t)=\frac{\exp \left(-\mathbf{k}^{2} l^{2} / 2\right)}{\left(1+i \hbar t / m l^{2}\right)^{\frac{3}{2}}} \exp \left[\frac{-\left(\mathbf{r}-i \mathbf{k} l^{2}\right)^{2}}{2 l^{2}\left(1+i \hbar t / m l^{2}\right)}\right] .
$$

We shall illustrate the influence of the shape of the Gaussian envelope on the motion of vortices in the simplest case of one rectilinear nonsqueezed vortex that is parallel to the $z$-axis and passes through the point $\left(x_{0}, 0\right)$ at $t=0$. The motion of such a line is composed of the free motion with the velocity $\mathbf{v}$ of the center of the Gaussian envelope and the motion induced by the shape of the envelope. The time-dependent wave function in this case is

$$
\frac{x(t)-x_{0}+i\left(y(t)-x_{0} \hbar t / m l^{2}\right)}{1+i \hbar t / m l^{2}} \psi_{\mathbf{k}}^{G}(\mathbf{r}, t) .
$$

The last term in the numerator represents the quantum correction to the free vortex motion and it vanishes in the limit, when the width of the Gaussian envelope $l$ tends to infinity. The speed of this additional motion depends on the smallest distance of the vortex line from the center of the Gaussian but does not depend on the motion of its center.

\section{VORTEX LINES FOR A PARTICLE IN A UNIFORM MAGNETIC FIELD}

In order to determine the motion of vortex lines in a magnetic field we need the counterpart of the generating function (18) in the presence of the field. We shall use the Schrödinger equation in the symmetric gauge

$$
\begin{aligned}
& i \partial_{t} \psi(\mathbf{r}, t)=\left[-\frac{\hbar^{2}}{2 m} \Delta\right. \\
& \left.-\frac{i \hbar e B}{m}\left(x \partial_{y}-y \partial_{x}\right)+\frac{e^{2} B^{2}}{8 m}\left(x^{2}+y^{2}\right)\right] \psi(\mathbf{r}, t),
\end{aligned}
$$

where the $z$-axis is taken along the magnetic field. The solution of this equation, that at the time $t=0$ has the form $\exp (i \mathbf{k} \cdot \mathbf{r}) \exp \left(-e B\left(x^{2}+y^{2}\right) / 4 \hbar\right)$ (a plane wave multiplied by the ground state Gaussian in the $x y$-plane), is

$$
\begin{aligned}
\psi_{\mathbf{k}}^{M}(\mathbf{r}, t) & =\exp \left(-e B\left(x^{2}+y^{2}\right) / 4 \hbar\right) \exp \left[-i \omega_{c} t / 2\right] \\
& \times \exp \left[\hbar\left(e^{-i \omega_{c} t}-1\right)\left(k_{x}^{2}+k_{y}^{2}\right) /(2 e B)\right] \\
& \times \exp \left[i\left(e^{-i \omega_{c} t}+1\right)\left(x k_{x}+y k_{y}\right) / 2\right] \\
& \times \exp \left[\left(e^{-i \omega_{c} t}-1\right)\left(x k_{y}-y k_{x}\right) / 2\right] \\
& \times \exp \left[i z k_{z}-i \hbar k_{z}^{2} /(2 e B)\right],
\end{aligned}
$$

where $\omega_{c}=e B / m$ is the cyclotron frequency. Since the differentiation with respect to the components of $\mathbf{k}$ brings down linear terms in the space variables, a single vortex along a straight line will preserve its form. All that can happen is the motion of this straight line. For example, a line vortex that at $t=0$ lies in the $y=a$ plane at an angle $\varphi$ to the field direction will move in time according to the following parametric representation

$$
\begin{aligned}
& y(x)=\frac{2 a+x \sin \left(\omega_{c} t\right)(1+\sin \varphi)}{1-\sin \varphi+(1+\sin \varphi) \cos \left(\omega_{c} t\right)} \\
& z(x)=-\frac{2 x \tan \varphi+a \sin \left(\omega_{c} t\right)(\sec \varphi+\tan \varphi)}{1-\sin \varphi+(1+\sin \varphi) \cos \left(\omega_{c} t\right)} .
\end{aligned}
$$


In Fig. 4 we show the motion of this line during one period of the cyclotron motion.

\section{VORTEX LINES FOR A PARTICLE IN A TRAP}

The study of vortex dynamics in a trap has a special significance because it may, perhaps, throw some light (as the first, linear approximation) on the behavior of the atoms that form the Bose-Einstein condensate. For simplicity, we shall consider a trap in the form of a spherically symmetric harmonic oscillator with the frequency $\omega$ and choose as $\phi_{0}(\mathbf{r})$ in the formula (14) the ground state wave function $\exp \left(-m \omega \mathbf{r}^{2} / 2 \hbar\right)$. The time dependent wave function that serves as a generator of vortex lines in this case is

$$
\begin{aligned}
\psi_{\mathbf{k}}^{T}(\mathbf{r}, t) & =\exp (-3 i \omega t / 2) \exp \left(-\frac{m \omega}{2 \hbar} \mathbf{r}^{2}\right) \\
& \times \exp \left(i e^{-i \omega t}\left(\mathbf{k} \cdot \mathbf{r}-\frac{\hbar \mathbf{k}^{2}}{2 m \omega} \sin (\omega t)\right)\right)
\end{aligned}
$$

As an example we shall choose the vortex line that at $t=0$ has the form of a circular ring of radius $R$ passing through the center. The wave function describing the time evolution of such a vortex is obtained by evaluating the appropriate derivatives of (34). Setting $\mathbf{k}=0$ after the differentiation, we obtain

$$
\begin{aligned}
{\left[e^{-2 i \omega t}\left(x^{2}+y^{2}-\frac{\hbar}{m \omega}\right)+\frac{\hbar}{m \omega}\right.} & \left.-e^{-i \omega t} R(2 x-i z)\right] \\
& \times \psi_{\mathbf{k}=\mathbf{0}}^{T}(\mathbf{r}, t)
\end{aligned}
$$

The time evolution of this vortex line is shown in Fig. 5 .

\section{VORTEX LINES IN RELATIVISTIC WAVE MECHANICS}

The description of vortex lines and their motion presented in this paper is not restricted to nonrelativistic wave mechanics based on the Schrödinger equation. It can be extended to a relativistic theory based on the Klein-Gordon equation. Let us consider a relativistic wave function $\phi$ of a free particle in the form of a plane wave

$$
\phi_{\mathbf{k}}(\mathbf{r}, t)=\exp (i \mathbf{k} \cdot \mathbf{r}) \exp \left(-i \omega_{\mathbf{k}} t\right)
$$

where $\omega_{\mathbf{k}}=c \sqrt{\mathbf{k}^{2}+(m c / \hbar)^{2}}$. This solution, in full analogy with the nonrelativistic case, may serve as a generating function for solutions with all kinds of vortex lines. For example, by taking first derivatives we generate a solution with one rectilinear vortex

$$
(x(t) \cos \chi+i y(t) \sin \chi) \phi_{\mathbf{k}}(\mathbf{r}, t) .
$$

The only difference between this solution and the nonrelativistic one (19) is due to a different relation between the velocity and the wave vector. In the relativistic case it is $\mathbf{v}=\nabla_{\mathbf{k}} \omega_{\mathbf{k}}=\hbar \mathbf{k} / \sqrt{(\hbar \mathbf{k} / c)^{2}+m^{2}}$, instead of just $\hbar \mathbf{k} / m$. Otherwise, the result is the same: the vortex line moves with the constant velocity $\mathbf{v}$. The motion of two vortex lines can also be determined in the same way as in the nonrelativistic case. In the limit, when $\mathbf{k} \rightarrow 0$ the relativistic case reduces to the nonrelativistic case, Eq.(25).

With the help of the generating function we may also determine the motion of a vortex ring in the relativistic theory. The wave function that determines the motion of the vortex ring in the relativistic case (the counterpart of the nonrelativistic wave function (21)) is

$$
\begin{aligned}
\psi^{C}(\mathbf{r}, t) & =\left[x(t)^{2}+y(t)^{2}-R^{2}\right. \\
+i a(z(t) & \left.\left.-\frac{2 \hbar\left(1-c^{2}\left(k_{x}^{2}+k_{y}^{2}\right) / \omega^{2}\right)}{a \sqrt{(\hbar \mathbf{k} / c)^{2}+m^{2}}} t\right)\right] \phi_{\mathbf{k}}(\mathbf{r}, t) .
\end{aligned}
$$

The speed of the vortex depends on the arbitrary parameter $a$ and may exceed the speed of light when $a$ is sufficiently small. This phenomenon, however, does not contradict the theory of relativity. It is quite similar to the so called superluminal propagation in optics [22,23, when the maximum of the signal travels with the velocity greater than $c$. In the present case it is the node of the probability density that may travel at an arbitrarily high speed.

The presence of vortex lines is not restricted to relativistic wave functions with only one component. They can also be found in wave functions with several complex components describing spinning particles. However, the freedom of attaching almost arbitrary vortex lines to any wave function is gone. There are stringent condition that must be satisfied in order to have physically admissible solutions. For example, a rectilinear vortex can be attached to a plane wave solution of the Dirac equation, but only when the direction of the vortex line is correlated with the particle momentum.

Of particular interest are the vortex lines associated with photon wave functions. Simple states of that category are the well known multipolar states: the eigenstates of the angular momentum. The corresponding photon wave functions have rectilinear vortices running from $-\infty$ to $\infty$, as in nonrelativistic wave mechanics. More elaborate vortex lines for photon wave functions are also possible but their study falls outside the scope of the present paper.

\section{CONCLUSIONS}

In our study of the motion of vortex lines in wave mechanics we have shown that this motion is determined by four elements: the shape of the vortex line, the shape of the wave function, the interaction between different vortex lines, and the external forces acting on the particle. 
Complete isolation of these effects is not possible but one can gain some insight by appropriate limiting procedures. We found that quantum corrections to the vortex motion are responsible for the mutual interaction between vortex lines and also for the vortex creation and annihilation. We believe that the qualitative features of the vortex motion will remain valid even in the much more complicated case of the mutually interacting particles, when the linear Schrödinger equation must be replaced by the nonlinear one.

\section{ACKNOWLEDGEMENT}

This work has been supported by the KBN Grant 2P30B04313.

[1] Aristotle, De Caelo (On the Heavens), Book II, Ch. XIII.

[2] R. Descartes, Principia Philosophiae, Amsterdam, 1644.

[3] W. Thomson (Lord Kelvin), Phil. Mag. 34, 15 (1867).

[4] G. Helmholtz, Crelles J. 55, 25 (18958) (English translation P. G. Tait, Phil. Mag. 33, 485 (1867)).

[5] P. G. Saffman, Vortex Dynamics (Cambridge University, Cambridge, 1992).
[6] D. R. Tiley and J. Tiley, Superfluidity and Superconductivity, 3rd ed. (Hilger, Bristol, 1990).

[7] F. Dalfolo and S. Stringari, Phys. Rev. A 53, 2477 (1996).

[8] D. S. Rokshar, Phys. Rev. Lett. 79, 2164 (1997).

[9] Y. Duan and H. Zhang, Eur. Phys. J. D 5, 47 (1999).

[10] A. L. Fetter, Phys. Rev. 151, 100 (1966).

[11] A. L. Fetter, in The Physics of Liquid and Solid Helium, edited by K. H. Bennemann and J. B. Ketterson, Part I (Wiley, New York, 1976).

[12] I. S. Aranson, A. R. Bishop, and L. Kramer, Phys. Rev. E 57, 5276 (1998).

[13] K.-P. Marzlin, W. Zhang, and E. M. Wright, Phys. Rev. Lett. 79, 4728 (1997).

[14] E. V. Goldstein, E. M. Wright, and P. Meystre, Phys. Rev. A 58, 576 (1998).

[15] O. Madelung, Z. Phys. 40, 342 (1926).

[16] S. K. Ghosh and B. M. Deb, Physics Reports 92, 1 (1982).

[17] I. Bialynicki-Birula and Z. Bialynicka-Birula, Phys. Rev. D3, 2410 (1971).

[18] I. Bialynicki-Birula, M. Cieplak, and J. Kaminski, Theory of Quanta (Oxford University Press, Oxford, 1992).

[19] H. Lamb, Hydrodynamics (Dover, New York, 1945), Ch. VII.

[20] T. Takabayasi, Prog. Theor. Phys. 14, 283 (1955); ibid. 70, 1 (1983).

[21] L. Janossy and M. Ziegler-Naray, Acta Phys. Hung. 20, 23 (1965).

[22] S. Chu and S. Wong, Phys. Rev. Lett. 48, 738 (1982).

[23] R. Y. Chiao, P. G. Kwiat, and A. M. Steinberg, Sci. Am. 269, 52 (1993). 

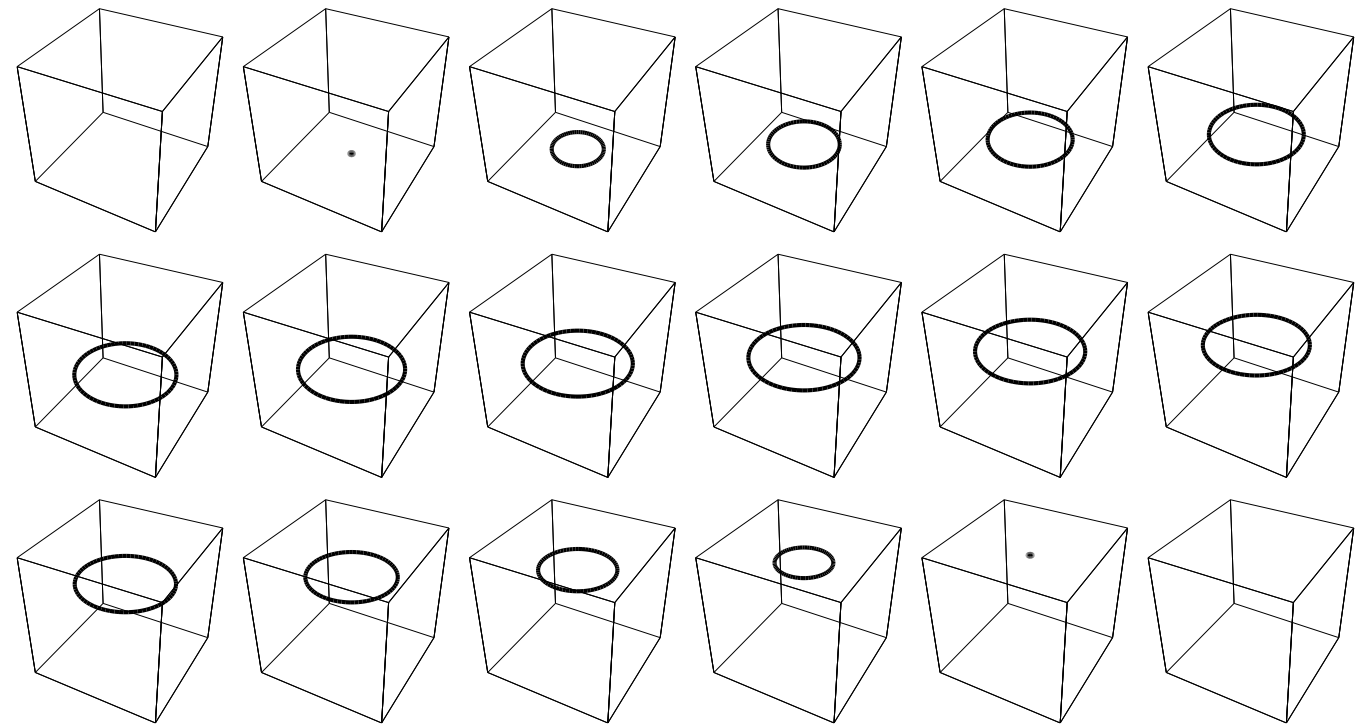

FIG. 1. Creation of a vortex at a point. A vortex ring is created at a point where the wave function vanishes.
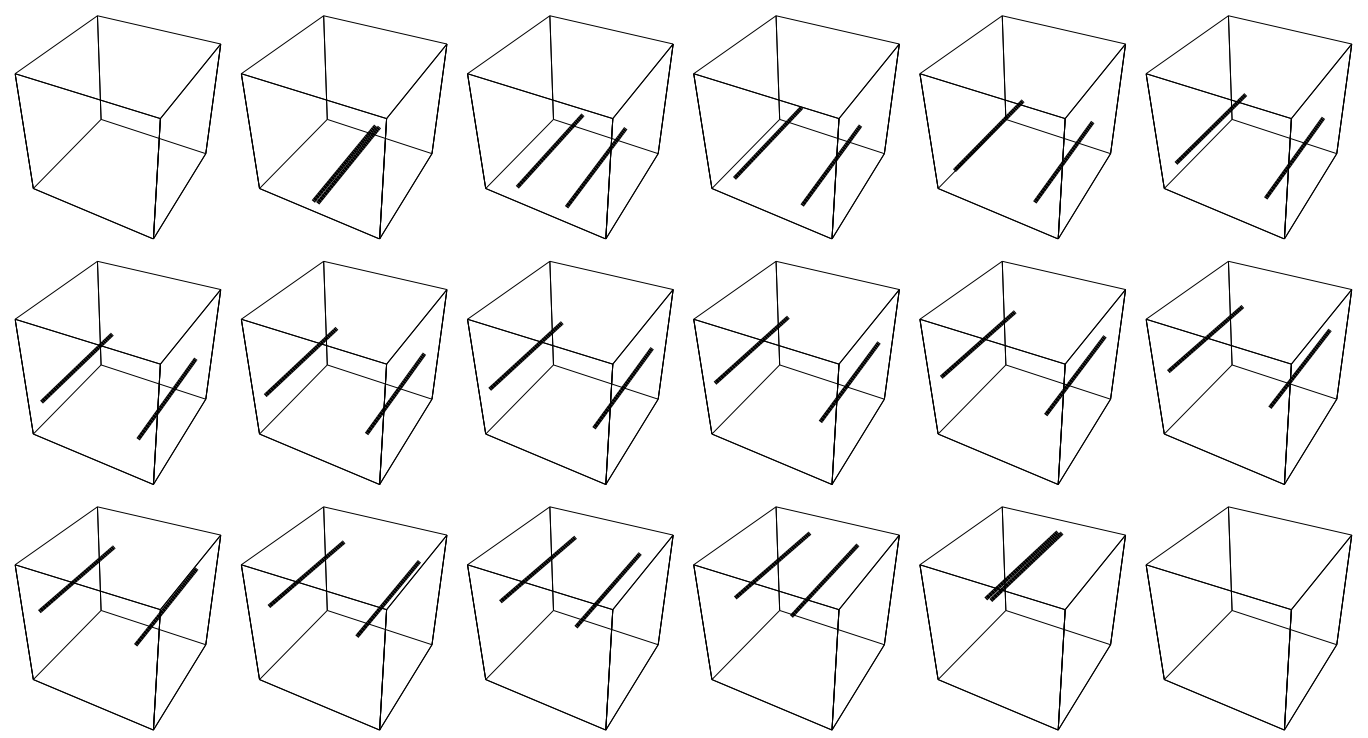

FIG. 2. Instantaneous creation and instantaneous annihilation of a vortex pair with opposite circulation along a line. 

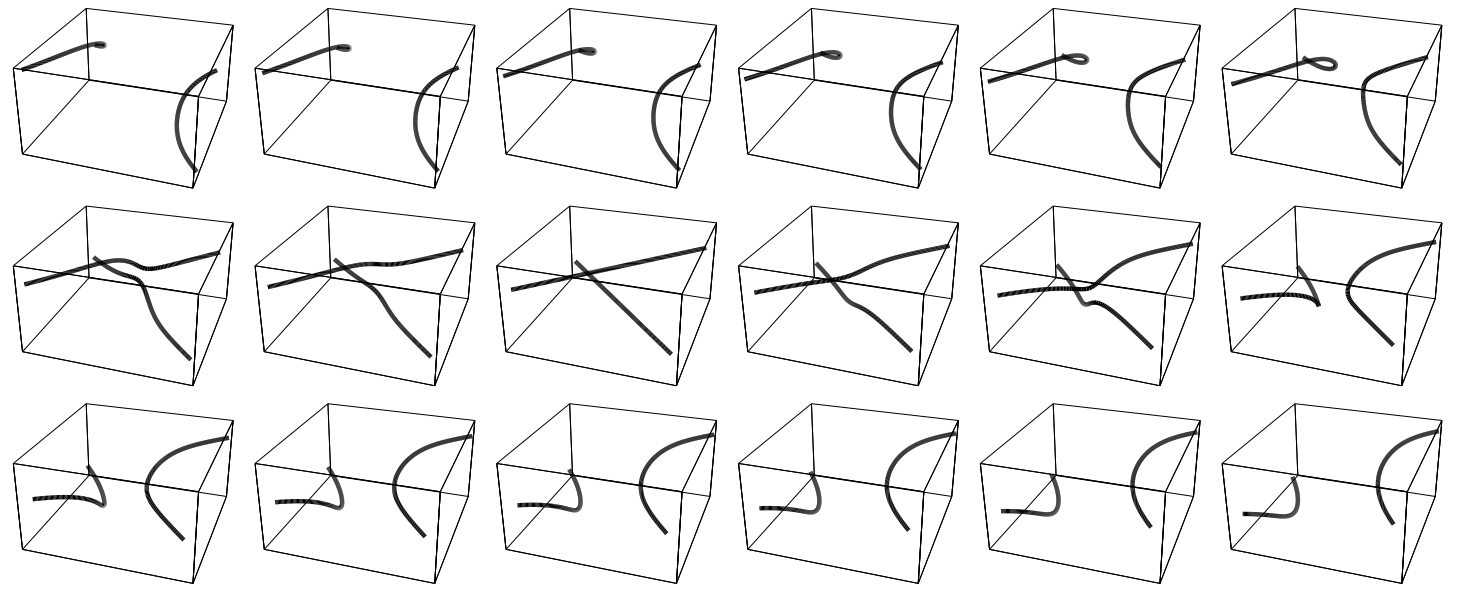

FIG. 3. Two vortex lines evolving in time from negative to positive values of $t$. At $t=0$ these vortex lines form a pair of nonintersecting straight lines.
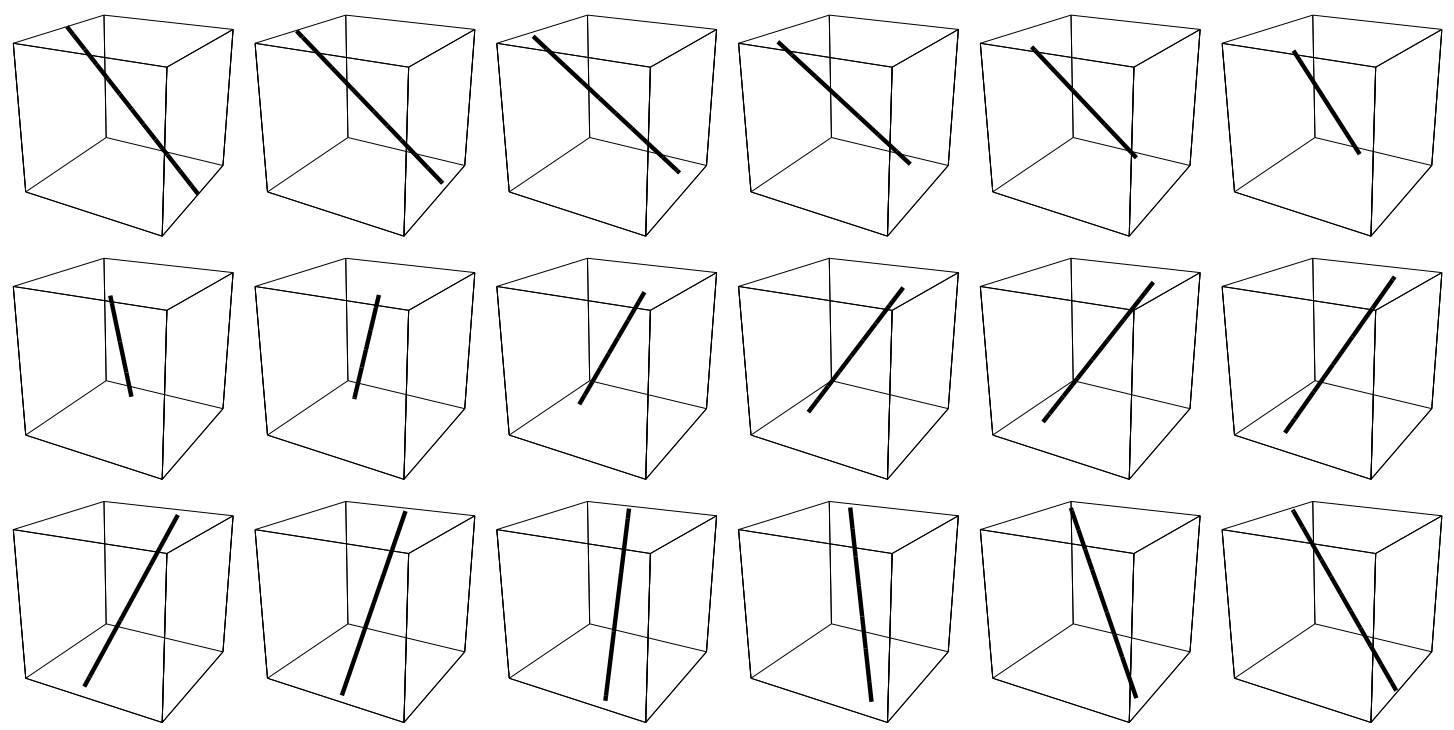

FIG. 4. Precession of a rectilinear vortex in a magnetic field. 

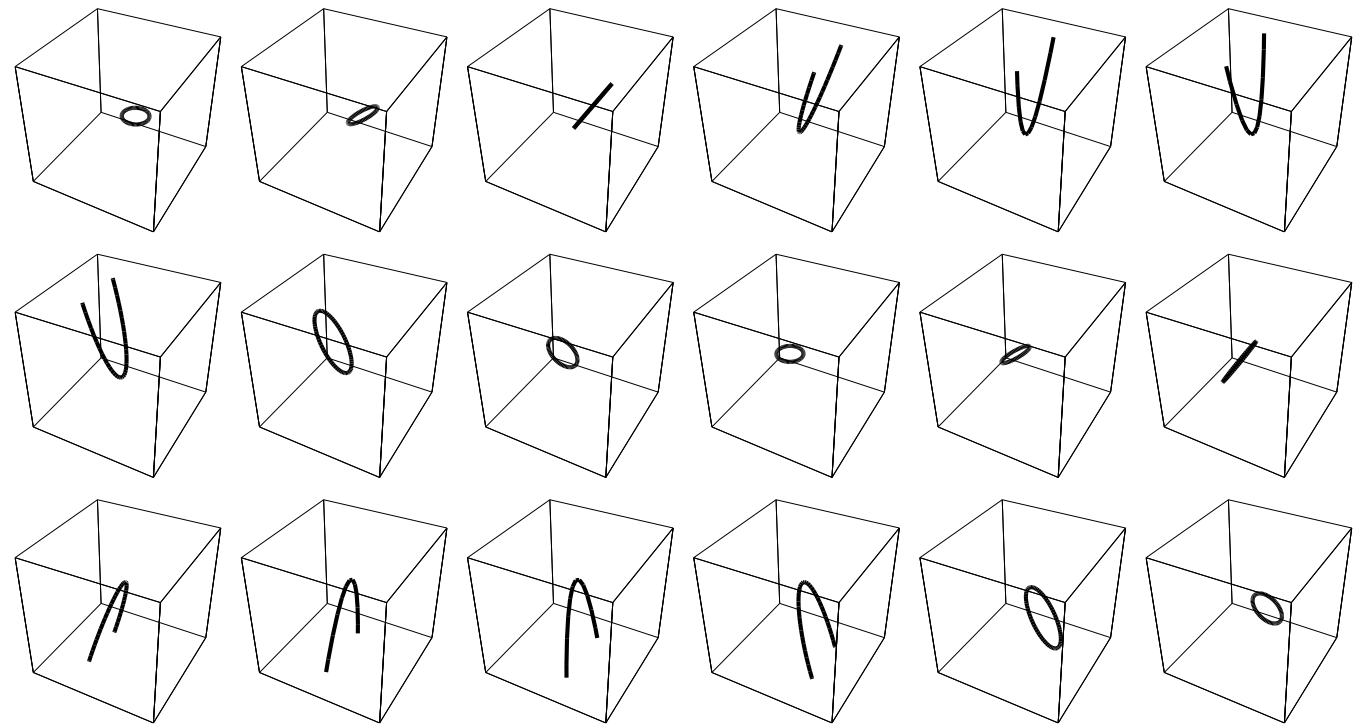

FIG. 5. Evolution of a vortex ring in a harmonic trap. 